\title{
Estudio de susceptibilidad a procesos de remoción en masa. Caso de estudio: Zona centro del Estado de Guerrero, México, mediante herramientas geotecnológicas
}

\author{
Wendy Romero-Rojas* \\ René Vázquez-Jiménez** \\ Rocío N. Ramos-Bernal**
}

Recibido 18 de agosto de 2018; aceptado 29 de octubre de 2018

\begin{abstract}
The processes of mass removal are recurrent geological processes and represent a latent threat in the State of Guerrero, due to its link whit extraordinary hydrometeorological events. In several studies that characterize these processes, Remote sensing techniques are applied, and the information is integrated through Geographical Information System (GIS). The model of susceptibility to mass removal processes included the interpretation of physical factors that intervene in these processes, which were characterized by different thematic maps. The final generation of the model consisted in the final accumulation of the individual contributions of each factor (Recondo, 2000; Hervas y Barredo, 2001: Hervas et. al., 2002), which represented a zoning by susceptibility index; the corroboration of the data was carried out through an inventory of lsndslides, generated through online image interpretation, applying the Google Earth tools. The results indicated that the model of susceptibility to mass

* Maestría en Ingeniería para la Innovación y Desarrollo Tecnológico, Facultad de Ingeniería (FI), Universidad Autónoma de Guerrero, Av. Lázaro Cárdenas s/n, CU, 39070, Chilpancingo, Guerrero, México, correo electrónico: geowromero@gmail.com

** Cuerpo Académico UAGro-CA-93 Riesgos Naturales y Geotecnología, FI, Universidad Autónoma de Guerrero, correos electrónicos: rvazquez@uagro.mx, rnramos@uagro.mx
\end{abstract}


removal processes allows to identify and effectively categorize the risk zone, in addition it was determined that the lithological, structural, topographic factors and the hydrometeorological events of September 2013, were the ones that caused the incidence of most recorded landslides.

Key words: landslides, conditioning factors, triggering detonating factors, Aster, susceptibility index.

\section{Resumen}

Los procesos de remoción en masa constituyen procesos geológicos recurrentes y representan una amenaza latente en el Estado de Guerrero, por su vínculo con eventos hidrometeorológicos extraordinarios. En varios estudios que caracterizan estos procesos, se aplican técnicas de percepción remota y la información se integra a través de los Sistemas de Información Geográfica. El modelo de susceptibilidad a procesos de remoción en masa, incluyó la interpretación de factores físicos que intervienen en dichos procesos, los cuales fueron caracterizados por distintos mapas temáticos. La generación final del modelo consistió en la acumulación final de los aportes individuales de cada factor (Recondo, 2000; Hervas y Barredo, 2001; Hervas, et al., 2002), el cual representó una zonificación por índice de susceptibilidad; la corroboración de los datos se realizó a través de un inventario de deslizamientos, generado a través de interpretación de imágenes on-line, aplicando las herramientas de Google Earth. Los resultados indican que el modelo de susceptibilidad a procesos de remoción en masa, permite identificar y categorizar efectivamente las zonas de riesgo, además se determinó que los factores litológicos, estructurales, topográficos y los eventos hidrometeorológicos de septiembre de 2013, fueron los que provocaron la incidencia de la mayoría de los deslizamientos registrados.

Palabras clave: deslizamientos, factores condicionantes, factores detonantes, Aster, indice de susceptibilidad.

\section{Resumo}

Os processos de remoção de massa constituem processos geológicos recorrentes e representam uma ameaça latente no Estado de Guerrero, por seu vínculo com eventos hidrometeorológicos extraordinários. Em vários estudos que caracterizam estes processos, se aplicam técnicas de sensoriamento remoto e a informação se integra através dos Sistemas de Informação Geográfica. O modelo de susceptibilidade a processos de remoção de massa, incluiu a interpretação de fatores físicos que interveem nestes processos, os quais foram caracterizados por distintos mapas temáticos. A geração final do modelo consistiu na acumulação final dos aportes individuais de cada fator (Recondo, 2000; Hervas y Barredo, 2001; Hervas, et al., 2002), que representou um zoneamento por índice de susceptibilidade; a corroboração dos dados se realizou através de um inventário de deslizamentos, gerado através de interpretação de imagens 
on-line, aplicando as ferramentas de Google Earth. Os resultados indicam que o modelo de susceptibilidades a processos de remoção de massa, permite identificar e categorizar efetivamente as zonas de risco, além disso se determinou que os fatores litológicos, estruturais, topográficos e os eventos hidrometeorológicos de setembro de 2013, foram os que provocaram a incidência da maioria dos deslizamentos registrados.

Palavras chave: deslizamentos, fatores condicionantes, fatores detonantes, Aster, indice de susceptibilidade.

\section{Introducción}

El presente trabajo se deriva de un proyecto de investigación desarrollado por Romero-Rojas (2017). Los eventos catastróficos de tipo hidrometeorológico ocurridos en México se han presentado cada vez con mayor frecuencia, evidenciado la amenaza y vulnerabilidad de la población. El cambio climático, la deforestación y los factores antropogénicos ligados al crecimiento poblacional y al uso del suelo en condiciones poco favorables han sido parteaguas en el incremento en la incidencia de estos fenómenos (González de Vallejo, 2002). Entre los eventos catastróficos más recurrentes en México están los procesos de remoción en masa, los cuales se convierten en riesgo latente cuando ponen en peligro a la sociedad.

En el Estado de Guerrero, Zúñiga-Gutiérrez et al. (2016) describen procesos de tipo deslizamiento en la comunidad de El Balsamar, municipio de Leonardo Bravo, en el 2002; en Taxco de Alarcón en las colonias de Martelas, La Molina y La Cadena, en el 2010. Estos fenómenos están íntimamente ligados a la precipitación registrada en eventos hidrometeorológicos extraordinarios.

En el año 2013 ocurrieron importantes procesos de remoción de masa, causados por el paso de la depresión tropical núm.13 en el Pacífico, frente a costas de Guerrero, iniciando a $170 \mathrm{~km}$ al suroeste de Tecpan de Galeana; posteriormente se desarrolló la tormenta tropical "Manuel", la cual propició entrada de humedad hacia el sur y occidente del país para pasar a la categoría de huracán. Simultáneamente se desarrolló en el Golfo el huracán "Ingrid”, afectando 29 estados del país (Bravo-Lujano, 2013). La lluvia registrada por estos fenómenos dieron lugar importantes deslizamientos, como el que ocurrió en La Pintada en Atoyac de Álvarez; donde se registraron 70 personas desaparecidas, 379 damnificados y 20 construcciones dañadas (Ramos-Bernal et al. 2015). Hubo también deslizamientos en las regiones de la montaña y centro del Estado, particularmente en los municipios de Leonardo Bravo y Chilpancingo.

Las herramientas integradoras para el análisis de los procesos de remoción en masa incluyen metodologías como la Percepción Remota, cuya función contribuye en el uso y manipulación de datos geográficos para la generación de modelos que, en 
conjunto con análisis estadísticos, permiten evaluar y gestionar el efecto negativo de los fenómenos naturales en beneficio de la sociedad. Estudios de este tipo, incluyen herramientas de análisis geográfico, geológico, ecológico y de infraestructura, que con el uso de Sistemas de Información Geográfica (SIG) como herramienta integradora, proveen de información oportuna y veraz que eventualmente puede servir en la toma de decisiones para establecer planes de prevención, atención o mitigación de riesgos naturales en escala regional.

\section{Zona de estudio}

Se localiza en la zona centro del Estado de Guerrero, entre los municipios de Chilpancingo de los Bravo, Leonardo Bravo, General Heliodoro Castillo y Coyuca de Benítez. Cubre una superficie de 128646 ha y está dentro de la provincia fisiográfica conocida como Sierra Madre del Sur (Morán-Zenteno, 1984) en la subprovincia cordillera costera del sur (INEGI, 2015). Los principales poblados incluidos en la zona son Izotepec, Yextla, Cruz de Ocote, Chautipan, Alcaparrosa, Inscuinatoyac, Coacoyulillo y Jaleaca de Catalán (Figura. 1).

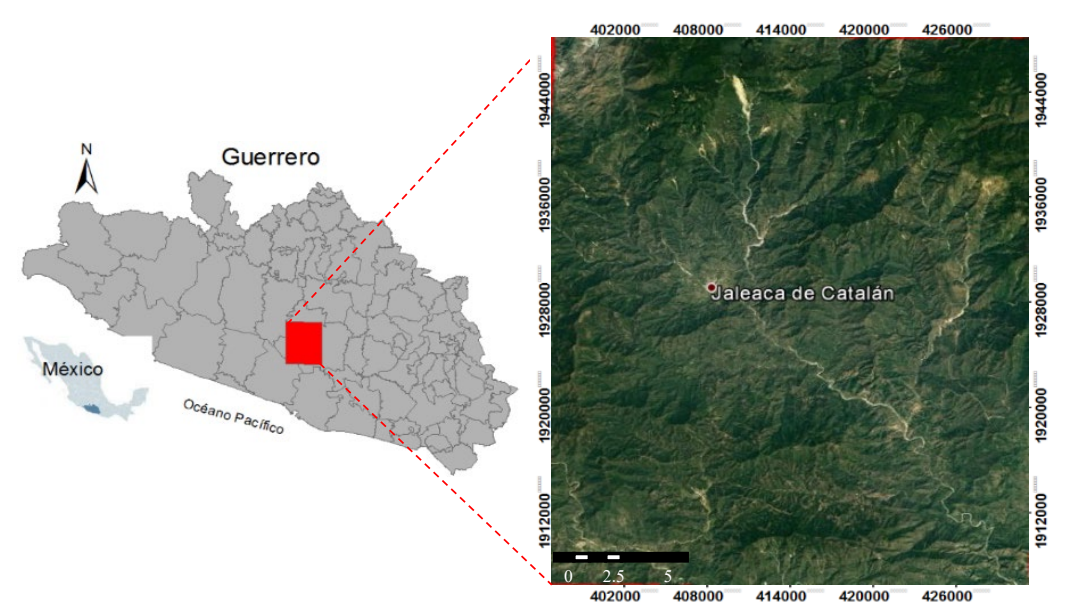

Figura 1. Zona de estudio.

\section{Materiales y métodos}

El trabajo contempla un análisis de cada uno de los factores que afectan la susceptibilidad a procesos de remoción en masa, mediante la interpretación de cartas geológico-mineras, topográficas y edafológicas y procesamiento digital de imágenes satelitales a través de los softwares ArcGis y Dinamica EGO. Las fechas de las imágenes usadas fueron de un año previo y otra posterior a los eventos climatológicos 
descritos, con la finalidad de identificar los procesos de remoción ocurridos por en el periodo.

Se integró un inventario de una muestra de deslizamientos, generado mediante herramientas de Google Earth que sirvieron como polígonos de entrenamiento y validación del modelo generado. Este proceso se realizó con la imagen del 12 de agosto de 2014, se identificaron y digitalizaron 70 polígonos con áreas mayores a $450 \mathrm{~m}^{2}$ que equivalen a dos pixeles en las imágenes Aster.

\section{Imágenes satelitales Aster}

Las imágenes satelitales usadas corresponden al sensor ASTER (Advanced Spaceborne Thermal Emission and Reflection Radiometer), de fechas 10 de diciembre de 2012 y 13 de diciembre de 2013. Las bandas de este sensor se conforman en tres subsistemas: el SWIR (región espectral del infrarrojo de onda corta); el TIR (región del infrarrojo térmico) y el VNIR (región espectral del infrarrojo cercano). Se eligieron las bandas 1,2 y $3 \mathrm{~N}$ cuya resolución espacial es de 15 metros.

Con estas imágenes se generó información adicional derivada, como los Índices de Vegetación, específicamente los NDVI (Índices de Vegetación de Diferencia Normalizada) y los Componentes Principales para ambas fechas de las imágenes (Figura 2).
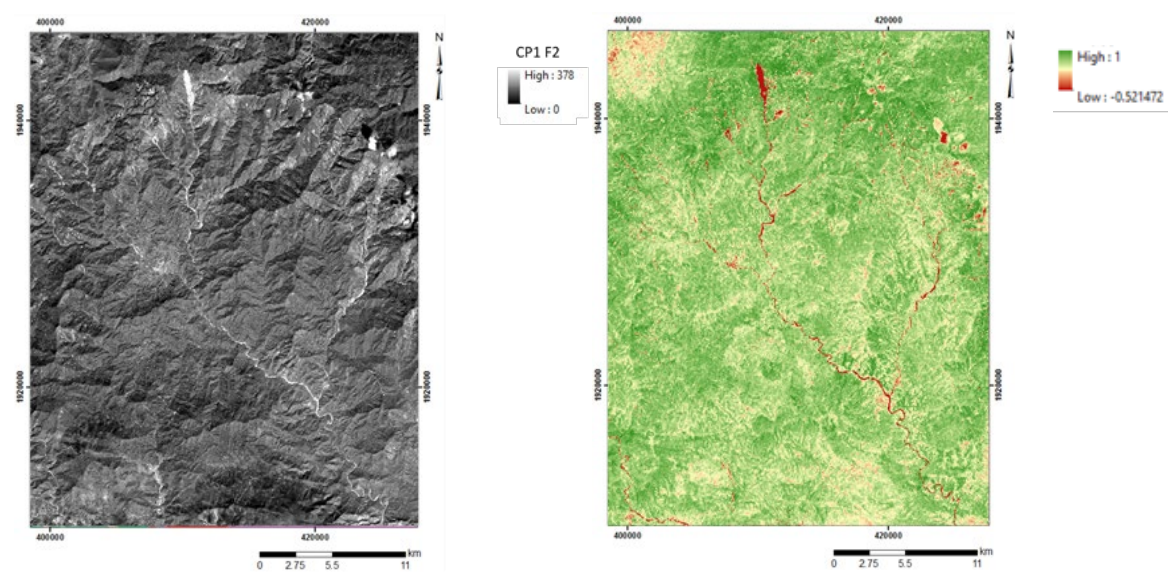

Figura 2. a) Componente Principal (CP). b) Índice de Vegetación de Diferencia Normalizada (NDVI) de fecha 13 de diciembre de 2013.

Posteriormente, a través del método de Regresión Lineal aplicado a las imágenes de NDVI y CP entre ambas fechas, se generó un modelo de predicción para determinar las variaciones existentes con los valores reales correspondientes, en cuyos casos 
los datos son expresados a través de mapas binarios categóricos (Romero-Rojas et al., 2017). La comparación de la información resultante sirvió para integración de un mapa de deslizamientos previos. El cual se usó como uno de los insumos del modelo de susceptibilidad propuesto.

\section{Mapas temáticos}

La interpretación y caracterización de los factores que intervienen en los procesos de remoción en masa, fueron representados por distintos mapas temáticos que alimentaron al modelo de susceptibilidad. El proceso consistió en identificar las variables que involucraron factores físicos, como la geología regional, el relieve, la edafología y la precipitación. Inicialmente, se realizaron tres mapas base: el mapa geológico, el Modelo Digital de Elevación y el edafológico (Figuras 3 a, b, c); en el último caso, sólo se requirieron los metadatos de la clasificación textural para su procesamiento posterior.

a)

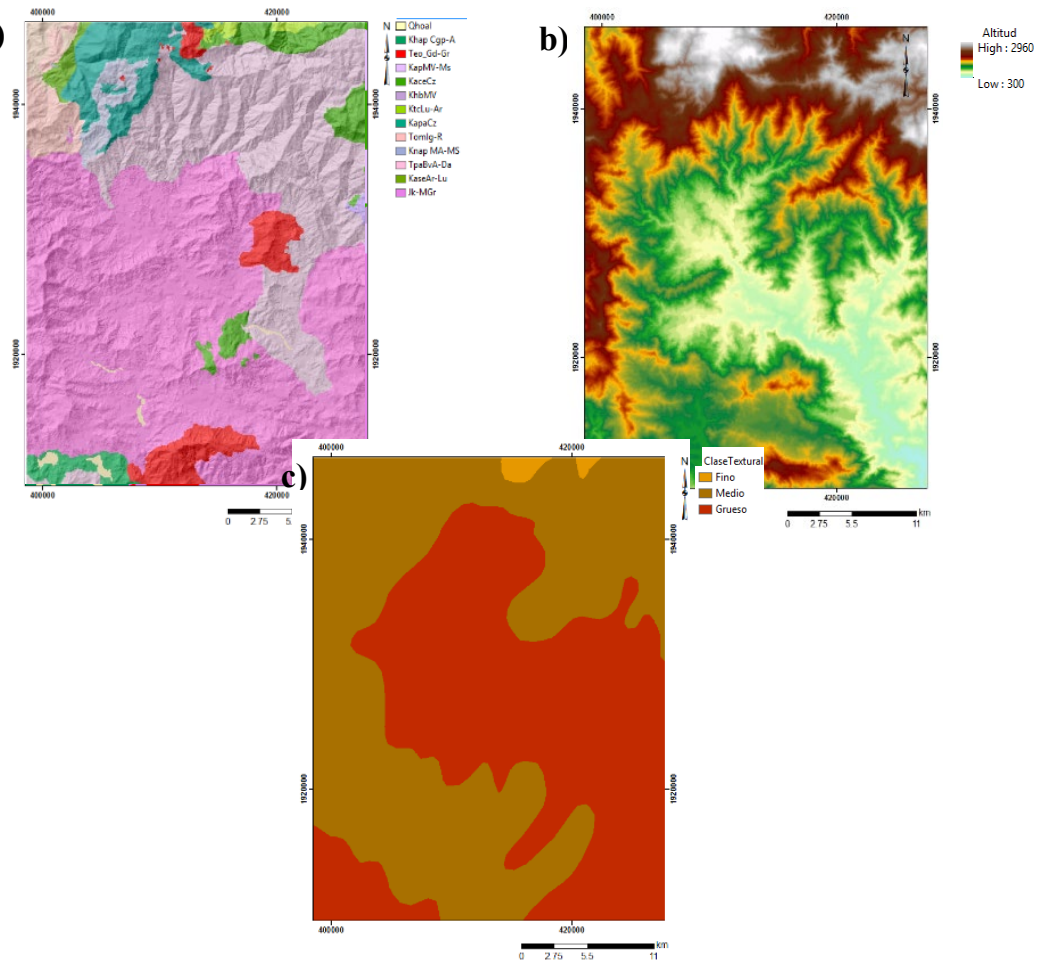

Figura 3. a) Mapa geológico, b) Modelo Digital de Elevación y c) Mapa edafológico (clasificación textural) del área de estudio. 
A continuación se muestra el resumen de los mapas temáticos y sus mapas finales que se incorporaron al modelo de susceptibilidad (Figura 4).

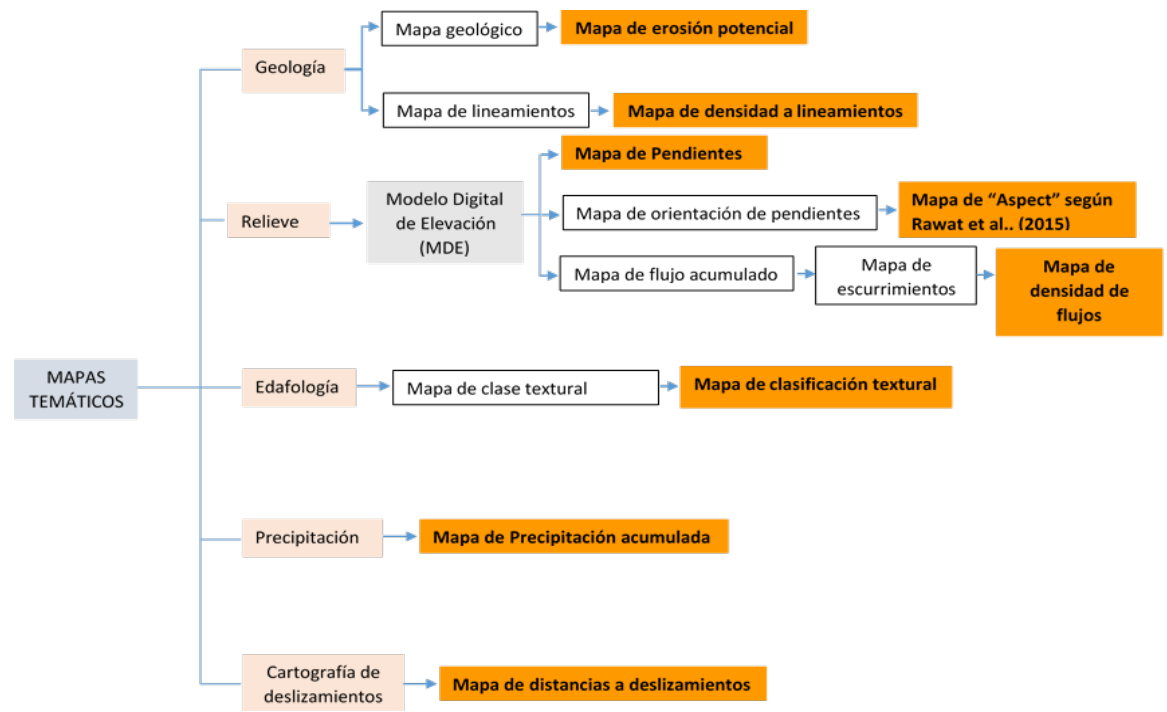

Figura 4. Resumen de mapas iniciales y su producto que servirán como insumos en el modelo de susceptibilidad a procesos de remoción en masa.

\section{Modelo de susceptibilidad a Procesos de Remoción en Masa (PRM)}

El modelo de susceptibilidad a PRM, consiste en el análisis de los factores causales de los PRM, evaluando para cada uno y pixel a pixel, su grado de aportación. Estas aportaciones individuales se acumulan en un índice global y de esta manera se integra la contribución relativa al peligro global por PRM que registra un área específica (Recondo, 2000; Hervas y Barredo, 2001; Hervas et al.; 2002).

\section{Normalización de los mapas temáticos}

Los mapas temáticos resultantes, representan las aportaciones individuales de cada uno de los factores considerados y registran valores tanto continuos como categóricos. Para hacer comparables las aportaciones, fue necesario normalizar la información estableciendo valores mínimos y máximos y así asignarle un valor normalizado a cada uno. Por conveniencia se definió que los mapas normalizados registraran valores entre 0 (aportación nula) ó 1 (aportación máxima.). El proceso de normalización para cada caso fue distinto, dependiendo del tipo de información que representan. Dicha normalización fue desarrollada con apoyo del software Dinamica EGO y se describen a continuación con mayor detalle. 


\section{Erosión potencial}

Los valores finales del análisis de erosión potencial dieron como resultado un mapa categórico de cinco categorías de aportación (1-muy baja a 5-muy alta). Con estos datos se obtuvo la relación de cada clase respecto al valor máximo (5). Los valores normalizados representan el grado de aportación del factor de erosión potencial en el modelo global de susceptibilidad (Tabla 1).

Tabla 1

Valores de normalización del mapa de erosión potencial

\begin{tabular}{lcc}
\hline \multicolumn{1}{c}{ Clase } & Normalización & Valores Normalizados \\
\hline 1 (muy bajo) & $1 / 5$ & 0.2 \\
2 (bajo) & $2 / 5$ & 0.4 \\
3 (medio) & $3 / 5$ & 0.6 \\
4 (alto) & $4 / 5$ & 0.8 \\
5 (muy alto) & $5 / 5$ & 1 \\
\hline
\end{tabular}

\section{Densidad a lineamientos}

El mapa de densidad a lineamientos tiene valores continuos dentro de un límite para este caso de $500 \mathrm{~m}$, establecido de acuerdo al promedio de la distancia de lineamientos a deslizamientos previos, de forma que pixeles fuera de este límite no aportaran al índice de riesgo global en el modelo de susceptibilidad, en lo que corresponde al factor de lineamientos.

El rango de valores del mapa de densidad va de 0 a 0.00254663 . Tomando el valor máximo como base para la normalización, a partir de la Ecuación 1 se obtienen la relación del valor de densidad de cualquier pixel respecto al valor máximo.

$$
\operatorname{Pn} i=\frac{P i}{V \cdot M}
$$

Donde:

Pni: Pixel normalizado $i$

$P i$ : Densidad de lineamientos del pixel $i$

V. M.: Valor máximo (0.00254663)

El rango de valores resultantes en el mapa normalizado es de 0 a 0.700423 y éste es el mapa que será usado en el modelo de susceptibilidad.

\section{Pendientes}

Para la normalización se analizó el histograma de frecuencias de pendientes para generar un umbral con base a la media más el doble de la desviación estándar (Vázquez- 
Jiménez, 2017) de los datos con el fin de identificar los valores más altos y tratarlos de la misma forma. La umbralización arrojó un valor final de $42^{\circ}$; de esta forma,

todos los pixeles iguales o mayores fueron considerados como máximos. Las Ecuaciones 2 a 4 sirvieron para normalizar las pendientes.

$S_{N}=0$ Para los pixeles con pendientes $\leq 5^{\circ}$ (Alcántara-Ayala, 2000)

$S_{N} i=\frac{S(i)}{\mu+2 \sigma}=L o s$ pixeles con pendientes $>5^{\circ} y \leq 42^{\circ}$

$$
S_{N} i=1 \text { Para los pixeles con pendientes }>42^{\circ}
$$

Donde:

$\mathrm{S}_{\mathrm{N}} i$ : Pendiente normalizada para el pixel $i$

$S i$ : Valor de la pendiente original para el pixel $i$

$\mu$ : Media de las pendientes

$\sigma:$ Desviación estándar de las pendientes

\section{Orientación de las pendientes}

Se identificaron las clases con mayor incidencia de deslizamientos de acuerdo al inventario; siguiendo la propuesta de Rawat et al. (2015); se categorizaron las orientaciones en ocho clases de orientaciones y para cada una se determinó el grado de susceptibilidad en funcion de la frecuencia de pixeles en el inventario. Finalmente, se reasignaron las clases con base al grado de susceptibilidad, donde el número uno corresponde al grado "bajo" y el número ocho al grado "muy alto".

Una vez asignadas las nuevas categorías; la normalización, consistió calcular la relación de cada clase respecto el valor mayor (8) de forma similar al caso de lineamientos.

\section{Densidad a flujos}

El mapa de densidad a flujos presenta valores continuos al igual que el mapa de lineamientos. Siguiendo la propuesta de Wang et al. (2017), el límite de influencia de flujos se estableció en $200 \mathrm{~m}$. El mapa original contiene valores desde 0 hasta 0.012571 . El proceso de normalización se obtuvo a partir de la relación del valor de la densidad de cada pixel y el valor máximo registrado (veáse Ecuación 1). De esta forma, el mapa de densidad de flujos normalizado registra valores entre 0 y 0.877316 .

\section{Efecto Potencial de la Precipitación (EPP)}

En el diseño del modelo se identificaron factores inherentes que requirieron ser combinados, con este argumento se procedió a construir un mapa que involucra a tres de los mapas temáticos: clase textural, pendientes (sin normalizar) y precipitación. En 
este sentido, se propuso la generación de un mapa que represente el efecto potencial de la precipitación combinando para cada pixel los valores de pendiente y la textura del suelo, calculado a partir de la Ecuación 5.

$$
\mathrm{EPP} i=P i * C T i * S i
$$

Donde:

EPPi: Efecto Potencial de la Precipitación para el pixel $i$

$P i$ : Precipitación Acumulada

$C T i$ : Clase Textural

Si: Pendiente

El mapa resultante se normalizó de la forma en que ha sido explicado y el mapa final registra valores entre 0 y 0.876719 .

\section{Modelo de Susceptibilidad a Procesos de Remoción en Masa}

De acuerdo con trabajos previos (Recondo, 2000; Hervas y Barredo, 2001; Hervas et al., 2002), la generación del modelo de susceptibilidad consistió en la acumulación pixel a pixel de la aportación individual de cada uno de los factores considerados expresados espacialmente a través de los mapas normalizados. De esta forma, el valor acumulado de las aportaciones individuales de cada factor representará el Índice de Susceptibilidad a Procesos de Remoción en Masa (ISPRM) y pueden ser expresados con la ecuación 6 .

$$
I S P R M=E P_{N}+D L_{N}+S_{N}+O S_{N}+D F_{N}+E P P_{N}
$$

Donde:

ISRPM: Índice de Susceptibilidad a Procesos de Remoción en Masa

$\mathrm{EP}_{\mathrm{N}}$ : Erosión Potencial Normalizada

$\mathrm{DL}_{\mathrm{N}}$ : Densidad a Lineamientos Normalizada

$\mathrm{S}_{\mathrm{N}}$ : Pendiente Normalizada

$\mathrm{OS}_{\mathrm{N}}$ : Orientación de las Pendientes (Aspect) normalizada

$\mathrm{DF}_{\mathrm{N}}$ : Densidad a Flujos Normalizada

$\mathrm{EPP}_{\mathrm{N}}=$ Efecto Potencial de la Precipitación Normalizada

El mapa resultante muestra valores entre 0.250 y 4.796 y fue categorizado en función del histograma, como se muestra en la tabla (Tabla 2): 
Tabla 2

Rango de valore para ISPRM y su categoría correspondiente

\begin{tabular}{ccc}
\hline No. & Rango ISPRM & Categoría \\
\hline 1 & $0.250-1.159$ & Muy bajo \\
2 & $1.159-1.690$ & Bajo \\
3 & $1.694-2.175$ & Medio \\
4 & $2.175-2.710$ & Alto \\
5 & $2.710-4.796$ & Muy alto \\
\hline
\end{tabular}

\section{Efecto de los remanentes de procesos de remoción antiguos}

Se desarrolló un análisis adicional, considerando como un factor condicionante más, la cercanía de los pixeles estables a deslizamientos antiguos. Para ello se obtuvo un mapa de distancias entre los pixeles sin procesos de remoción y zonas con la presencia de ellos. Dicho mapa resulta con valores mínimos de 15 metros (por la resolución espacial de las imágenes), siendo estos los pixeles inmediatos a zonas deslizadas y por ello los más susceptibles a la ocurrencia de nuevos procesos.

De acuerdo a este análisis y con el fin de normalizar el mapa de distancias con valores entre 0 y 1 , para incorporarlo al modelo de ISPRM y explorar su efecto, se aplicó la Ecuación 7.

$$
D N i=\frac{R E}{D i}
$$

Donde:

$D N i$ : Distancia Normalizada del pixel $i$

$R E$ : Resolución espacial $(15 \mathrm{~m})$

$D i$ : Distancia a un deslizamiento del pixel $i$

Una vez obtenidos los valores normalizados correspondientes a cada pixel, se ajustó a la ecuación inicial del modelo (5) incorporando la distancia a deslizamientos, tal como lo muestra la siguiente ecuación:

$$
I S P R M=E P_{N}+D L_{N}+S_{N}+O S_{N}+D F_{N}+E P P_{N}+D N_{N}
$$

Donde:

ISRPM: Índice de Susceptibilidad a Procesos de Remoción en Masa

$\mathrm{EP}_{\mathrm{N}}$ : Erosión Potencial Normalizada

$\mathrm{DL}_{\mathrm{N}}$ : Densidad a Lineamientos Normalizada

$\mathrm{S}_{\mathrm{N}}$ : Pendiente Normalizada

$\mathrm{OS}_{\mathrm{N}}$ : Orientación de las Pendientes (Aspect) normalizada

$\mathrm{DF}_{\mathrm{N}}$ : Densidad a Flujos Normalizada

EPP $_{\mathrm{N}}$ : Efecto Potencial de la Precipitación Normalizada

$\mathrm{DN}_{\mathrm{N}}$ : Distancia Normalizada a Deslizamientos antiguos 


\section{Evaluación del modelo de susceptibilidad y su relación con las localidades del área de estudio}

Como evaluación final del modelo resultante, se generó una traslape de los polígonos correspondientes a las poblaciones de la zona y para explorar su relación con las zonas susceptibles a los procesos de remoción en masa, con la finalidad de identificar zonas potenciales de riesgo a sufrir algún proceso de remoción que represente una amenaza a la población.

\section{Resultados}

\section{Mapa de erosión potencial}

Hacia la parte centro-sur del área de estudio se aprecia una erosión potencial 'muy baja' seguido por el grado 'medio' y ‘bajo' hacia la parte centro-norte, evidenciando que la escorrentía superficial en condiciones normales no afecta directamente el comportamiento mecánico de las rocas (Figura 5a).

De acuerdo al inventario, los principales deslizamientos se presentaron en formaciones que exhiben filitas, metaandesitas, pizarras, rocas volcánicas intemperizadas, calizas y lutitas, lo que evidencia que las características físicas superficiales de las rocas presentan fracturamiento, planos de foliación dominantes o estratificación, intemperismo y contacto litológico de las unidades, lo que influenciaron a la inestabilidad del terreno aunado a otros factores superficiales propicios para la generación de PRM.

\section{Mapa de densidad a lineamientos}

En el mapa se observan lineamientos únicos y otros interceptados hasta ocho veces con mayor incidencia al oeste de la zona. De acuerdo al inventario, puede observarse que la mayoría de los PRM son atravesados por, o están dentro del rango asignado como zona de material vulnerable, específicamente hacia la parte noreste (Figura 5b).

a)

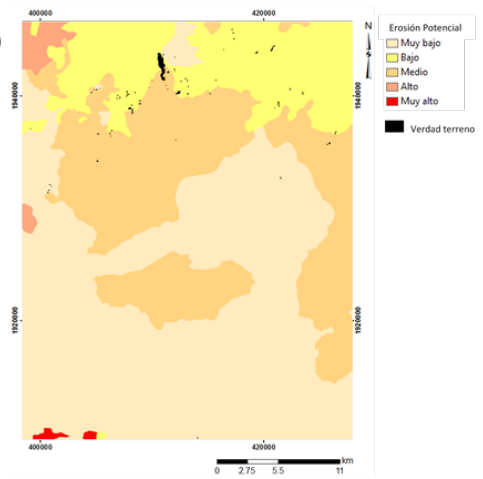

b)

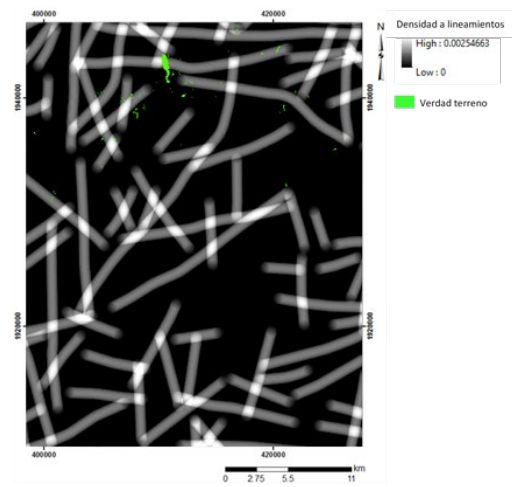

Figura 5. Mapas temáticos finales: a) Erosión potencial y b) Densidad a lineamientos. 


\section{Mapa de pendientes}

El análisis realizado con los polígonos del inventario indican que la mayoría de ellos se distribuyen en las pendientes que oscilan entre $\operatorname{los} 25^{\circ}$ y $33^{\circ}$, lo cual indica que las zonas con pendientes similares pueden ser susceptibles a PRM (Figura 6a).

\section{Mapa de orientación de las pendientes}

De acuerdo al inventario, se observa que los deslizamientos se presentaron en laderas orientadas al sureste, sur y suroeste, lo cual indica que los PRM no solo están controlados por la orientación de los flancos de las geoformas sino que podrían incluirse la intensa foliación y la litología presente en la zona (Figura 6b).
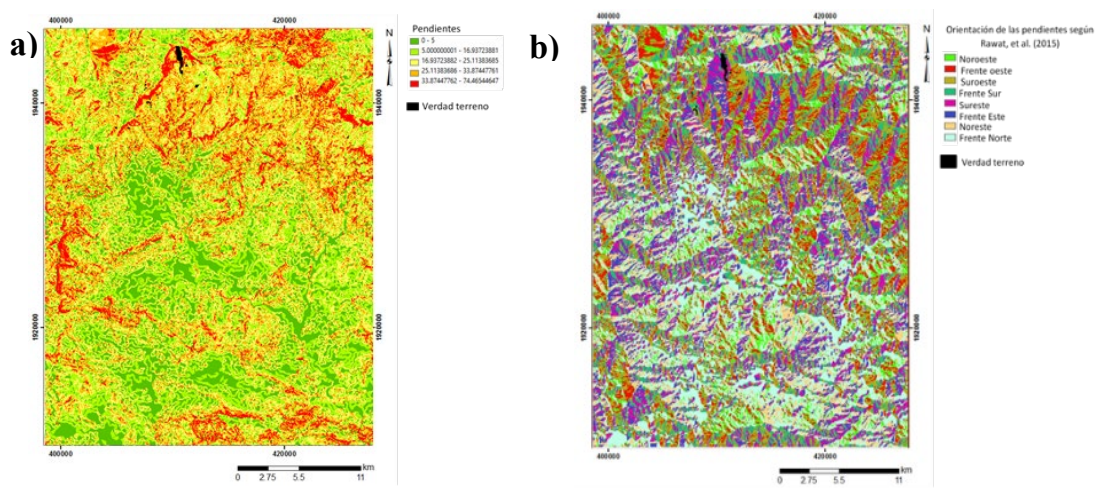

Figura 6. Mapas temáticos finales: a) Pendientes y b) Orientación de las pendientes.

\section{Mapa de densidad a flujos}

Los flujos presentan continuidad con la distribución de los rasgos estructurales de la zona. Según el inventario, en los lugares de deslizamientos se observan uniones de cauces principales y secundarios o cercanía a ellos. Con esto, la distribución espacial de los flujos y en conjunto con factores externos condicionan la generación de PRM en la zona de estudio (Figura 7a).

\section{Mapa de clasificación textural}

Según este mapa, el material posee alta capacidad de filtración. Los polígonos del inventario se encuentran distribuidos en las zonas de clases media y alta. Al existir diferencia textural entre materiales adyacentes y una escorrentía superficial que se infiltra, el material se vuelve inestable, afectando no solo a la zona de transición sino que también vulnera el material circundante (Figura 7b). 
a)

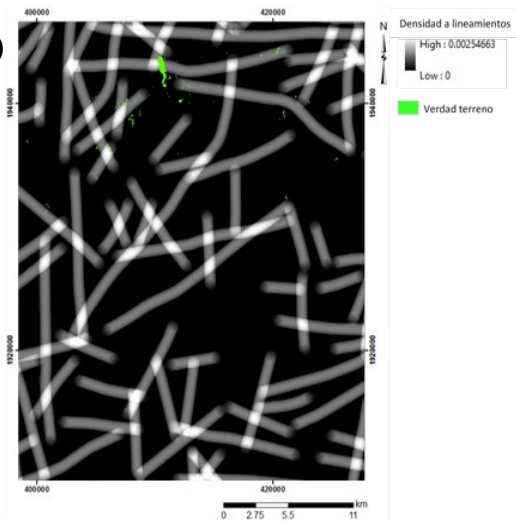

b)

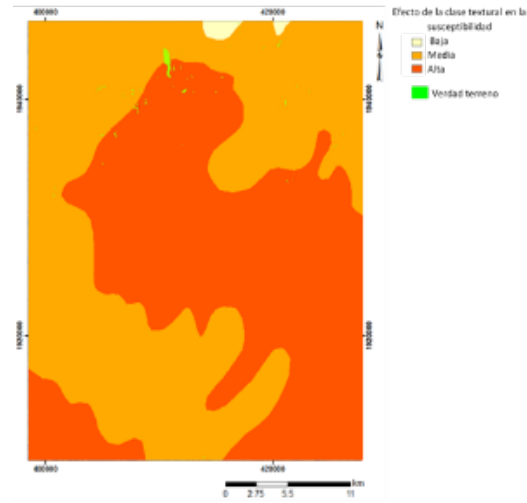

Figura 7. Mapas temáticos finales: a) Densidad Figura 25, Mapa de clase textural

\section{Mapa de precipitación acumulada}

Según este mapa, la menor precipitación se localizó al noroeste y sur; mientras que la mayor precipitación se dio al Noreste, esto debido a la presencia de la serranía sur, lo que favorece una mayor cantidad de precipitación debido al ascenso y enfriamiento adiabático del aire por la presencia de vientos húmedos (García-Amaro de Miranda, 2003).

La distribución de los polígonos del inventario se encuentran entre los valores medios-altos de precipitaciones, lo cual indica alta influencia de este factor para la ocurrencia de los PRM (Figura 8).

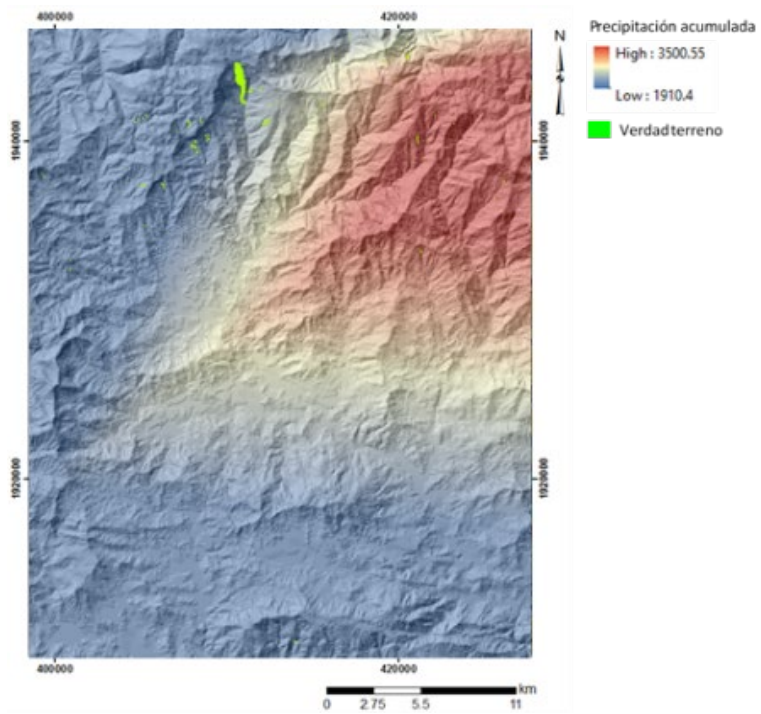

Figura 8. Mapa temático final de Precipitación acumulada. 


\section{Mapa de distancia a deslizamientos}

Los mapas categóricos resultantes del proceso de detección de cambios a partir de las imágenes del proceso de detección de cambios a partir de las imágenes NDVI y CP1 indican que el mapa de deslizamientos generado con los datos NDVI tiene mayor índice de aciertos y menor índice de errores, con un índice global de concordancia Kappa de $86 \%$. Por otro lado, el mapa de deslizamientos generado con datos de CP!, presenta un índice Kappa de $80.6 \%$.

En la Figura 8e y 8f, es posible observar a detalle las diferencias en la delimitación automática de los deslizamientos aplicando la Regresión Lineal, para ambos tipos de datos (NDVI y CP1). Además se observa una mayor cobertura los polígonos detectados como deslizamientos usando NDVI (en amarillo en las Figuras 9d, 9e y 9f) respecto a los polígonos detectados como deslizamientos usando CP1 (en verde en Figuras 9a, 9b y 9c).

En las zonas de traslape con los polígonos del inventario (Figuras 9c y 9f) se observa en general que ambos resultados corresponden con los polígonos del inventario. Sin embargo, los deslizamientos detectados usando imágenes NDVI presentan visualmente mayor precisión en el ajuste de los bordes de los deslizamientos detectados.
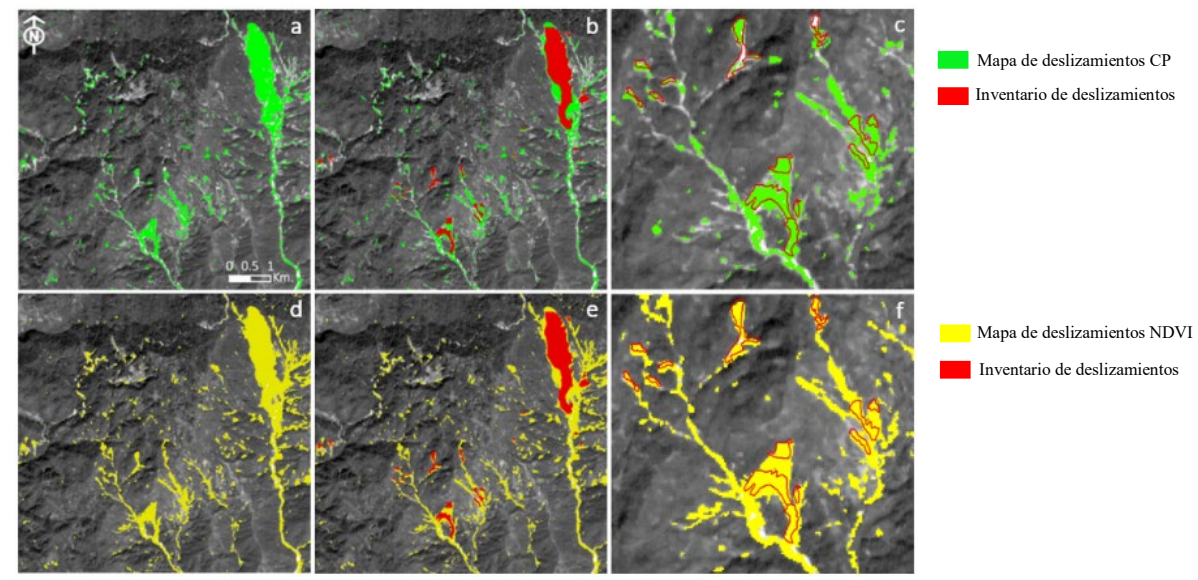

Figura 9. Acercamiento a zonas de los mapas de deslizamientos generados a partir de datos CP-verde y NDVI-amarillo (a y d). Traslape de inventario de deslizamientos en rojo sobre deslizamientos detectados por el modelo (b y e). Traslape de inventario de deslizamientos (c y f).

\section{Modelo de susceptibilidad a procesos de remoción en masa}

Según la información de los pixeles, las categorías de susceptibilidad media y baja son predominantes en el área de estudio y se localizan en la región centro-sur. Las 
rocas presentes corresponden en menor superficie a filitas, metaandesitas y pizarras hacia la parte este y suroeste; gneises y granitos milonitizados, estas últimas hacia la parte central y representan mayor extensión de área (Figura 10).

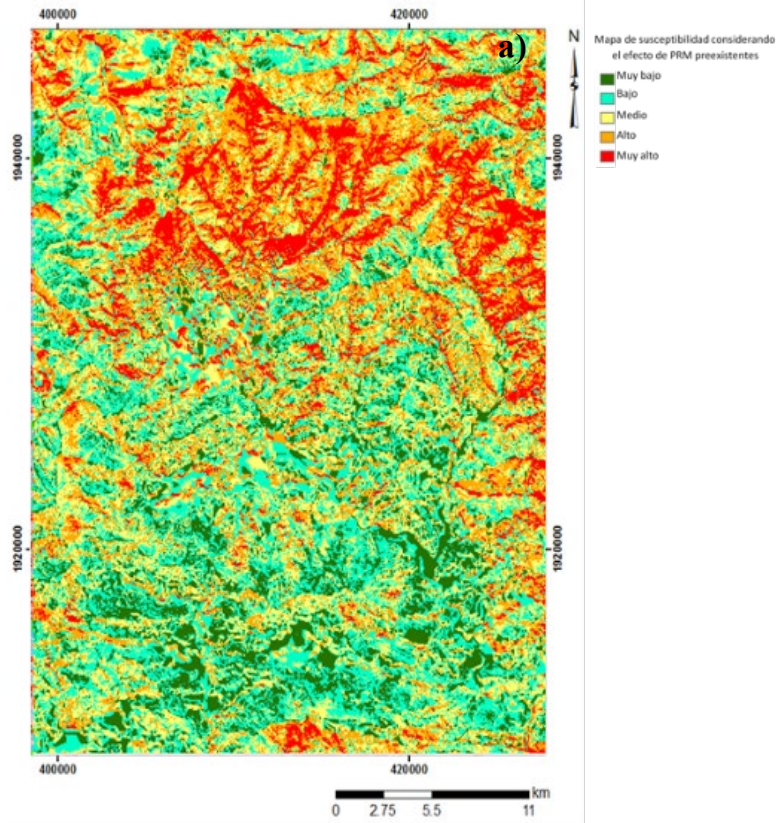

Figura 10. Mapa de susceptibilidad a procesos de remoción en masa (PRM).

Las rocas graníticas presentan mayor resistencia a procesos de meteorización e impermeabilidad a procesos hidrológicos, por tanto, son poco susceptibles a erosión por escorrentía superficial, lo que limita la presencia de PRM.

Las zonas de susceptibilidad alta y muy alta están al norte y noreste, donde existen pendientes pronunciadas y unidades litológicas poco competentes, como metaandesitas, filitas y pizarras y al este hay afloramientos de brechas y rocas volcánicas. La mayoría de las rocas muestran contenido de micas las cuales en estado de meteorización producen suelos ricos en arcillas con gran capacidad de retención de agua y baja resistencia mecánica (Alcántara-Ayala, 2000), tal es el caso de las filitas y pizarras cuya extensión es predominante en las áreas de susceptibilidad alta y muy alta.

Las áreas hacia el sur con susceptibilidad alta, se relacionan con rocas competentes a la erosión y escorrentía superficial, pero están en una zona de contacto litológico, lo cual debilita la roca, además se observa una transición en el grado textural de medio a alto. La clasificación textural juega un papel importante debido a la presencia 
de rocas permeables, es decir, con espacios intersticiales capaces filtrar el agua a diferencia de material fino.

Los rasgos estructurales muestran una relación importante con las zonas de alta y muy alta susceptibilidad, los lineamientos que atraviesan estas zonas contienen más de una intersección con otros lineamientos; asimismo los flujos presentes muestran influencia de los valores más altos hacia las zonas de alta y muy alta susceptibilidad, además de que forman parte de la escorrentía superficial.

Además de lo anterior, la precipitación acumulada en esas fechas jugó un rol importante en los procesos de remoción, ya que en estas zonas se registró la mayor concentración pluvial con valores que oscilan entre los $2000 \mathrm{~mm}$ y $3500.55 \mathrm{~mm}$, esta última la de mayor precipitación histórica registrada en la zona

Las zonas con mayor potencial a PRM, pueden ser corroborados a través de la ubicación espacial de los polígonos del inventario de deslizamientos (verdad terreno). Se aprecia su distribución hacia las zonas con los valores de mayor susceptibilidad, asimismo el deslizamiento de mayor tamaño es ejemplo de un proceso de remoción que cumple con la presencia de los factores condicionantes y detonantes para su generación: presencia de roca poco coherente, fracturada y foliación en distintas direcciones, con una pendiente pronunciada mayor a $33^{\circ}$, interacción de un flujo que atraviesa longitudinalmente el área con aportación de agua significativo. La parte estructural indica que la orientación de uno de los lineamientos cruza perpendicularmente al deslizamiento, lo cual es un factor importante en el desarrollo del movimiento.

Los valores resultantes al incluir como factor a los deslizamientos previos, indican que la existencia de remanentes de PRM influye e incrementa la susceptibilidad, ya que el material no se encuentra estable y las condiciones físicas y mecánicas no son iguales al momento de su depósito. Inicialmente, los polígonos del inventario se veían comprometidos en las áreas de muy alta susceptibilidad, pero sus contornos no, además de que ocasionalmente se encontraban en áreas de susceptibilidad menores (Figuras 11a y b). En el modelo de susceptibilidad que involucra antiguos deslizamientos, se aprecia un cambio importante en la distribución espacial de los polígonos, ya que los incluye directamente con las áreas de mayor susceptibilidad, lo que evidencia un mayor control de las áreas propensas a PRM (Figuras. 11c y d).

Según esto, es importante la incorporación de los PRM preexistentes, al hacerlo se están considerando variables que pudiesen omitirse en el modelo inicial, por tanto, asume una mejora considerable en la detección de zonas vulnerables a procesos de remoción en masa. 

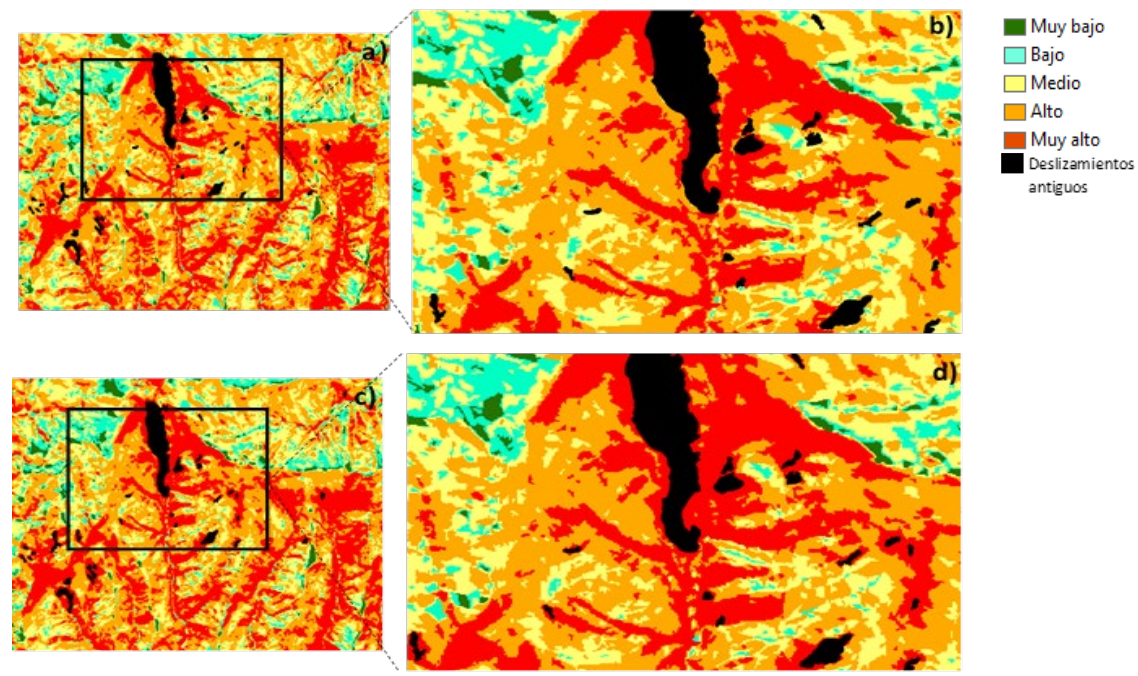

Figura 11. Comparativos del modelo de susceptibilidad inicial (a), el modelo con PRM preexistentes (c). Se incluyen polígonos de deslizamientos (en color negro).

\section{Evaluación del modelo de susceptibilidad y su relación con las localidades del área de estudio}

Se localizan nueve poblados dentro del área de estudio, de los cuales Ixotepec, Yextla, Colorada y Jaleaca de Catalán representan las localidades de mayor población con un total de 6970 habitantes (SEDESOL, 2013). Según el modelo de susceptibilidad que involucra a los deslizamientos antiguos, las tres clases de mayor riesgo (medio, alto y muy alto) se encuentran presentes en estas zonas, predominantemente la clase de susceptibilidad media y alta en los poblados de Ixotepec y Yextla; mientras que en la porción centro-sur, la clase predomínate es la media y alta en los poblados de Jaleaca y Colorada (Figura 12). Es importante recalcar la importancia del impacto de los resultados de este trabajo, las posibles consecuencias directas en los asentamientos y el futuro planeamiento territorial.

\section{Conclusiones}

La aplicación de herramientas geotecnológicas, permitió el análisis de los factores (condicionantes y detonantes) considerados como el modelo de susceptibilidad y la representación de sus valores expresados espacialmente.

El desarrollo del modelo de susceptibilidad a procesos de remoción en masa aplicando técnicas de Percepción Remota y SIG, permitió identificar las zonas susceptibles de acuerdo a los valores de los factores detonantes y condicionantes y a los 
procesos de remoción anteriores; siendo el tipo de roca, específicamente filitas, pizarras, lavas, brechas y las discontinuidades inherentes a ellas, es decir, intensa foliación y clase textural; los lineamientos, las pendientes de las laderas y la precipitación acumulada asociada a los eventos hidrometeorológicos extraordinarios registrados en 2013, los principales factores que provocaron la incidencia de los deslizamientos en la zona de estudio.

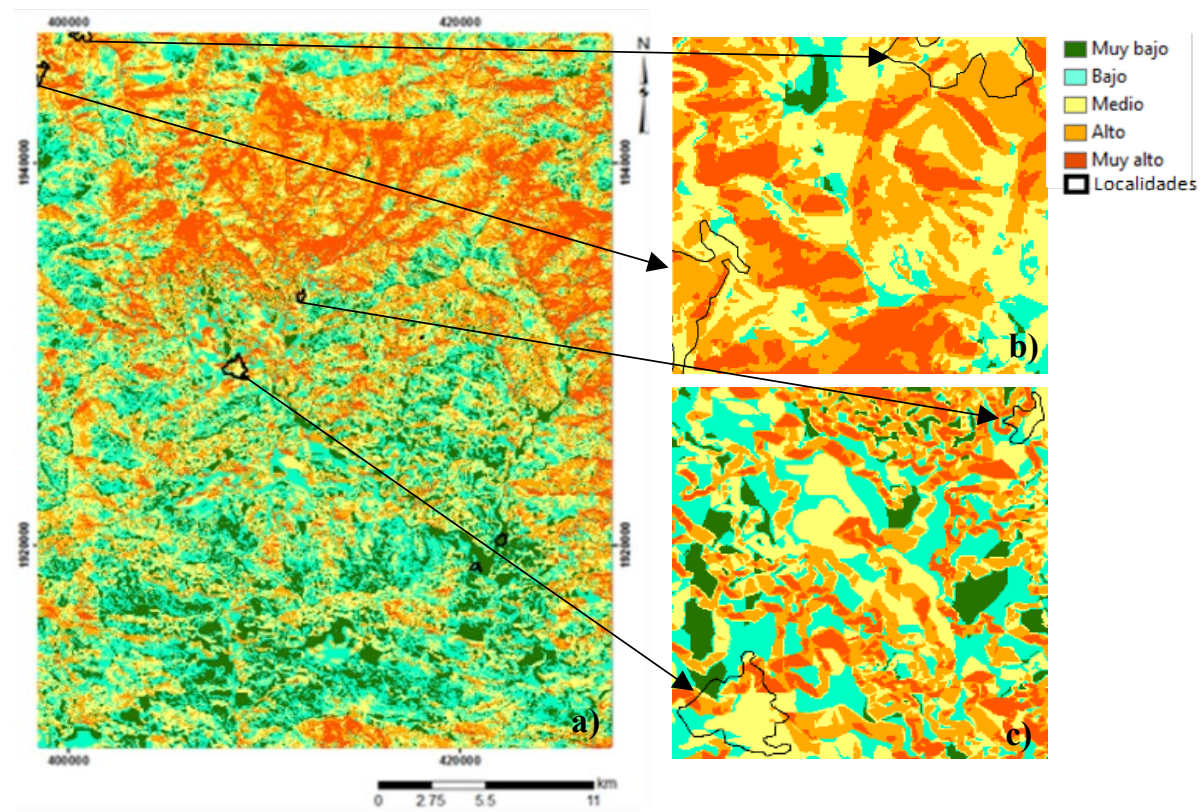

Figura 12. Poblados del área de estudio y su relación con las clases susceptibilidad. a) Modelo de susceptibilidad; b) Poblados de Yextla (polígono sup. der.) e Izotepec (polígono inf. izq.); c) Poblados de Colorada (polígono sup. der.) y Colorada (polígono inf. izq.).

Estudios como el presente, puede apoyar a distintas instituciones encargadas de salvaguardar a la población en eventos de este tipo, localizando las zonas donde se registra una mayor susceptibilidad de acuerdo a los factores considerados e integrando planes de atención o prevención a desastres considerando zonas con mayor prioridad que otras. Es importante mencionar también que esta investigación, puede complementarse aún más con la definición de rutas de evacuación, identificación de centros de atención y capacidad de albergues, etc.; todo ello poniendo como eje fundamental el conocimiento del territorio a través de la aplicación de las tecnologías de la información geográfica. 


\section{Bibliografía}

Alcántara-Ayala, I. (2000). "Índice de susceptibilidad a movimientos de terreno y su aplicación en una región semiárida", Revista Mexicana de Ciencias Geológicas, vol. 17, núm. 1, Instituto de Geología, Universidad Nacional Autónoma de México, México, pp. 66-74.

Bravo-Lujano, C., (2013). "Reseña del huracán 'Manuel' del Océano Pacífico. CONAGUA-SMN", 9 pp. Disponible en: $<$ http://smn.cna.gob.mx/tools/DATA/ Ciclones\%20Tropicales/Ciclones/2013-Manuel.pdf $>$, consultado el 10 de noviembre de 2016.

García-Amaro de Miranda, E. (2003). "Distribución de la precipitación en la República Mexicana", Investigaciones Geográficas, Boletín del Instituto del Instituto de Geografia, núm. 50, UNAM, pp. 67-76. Disponible en: $<$ http://www.scielo.org.mx/pdf/igeo/n50/n50a9.pdf $>$, consultado el 3 de noviembre de 2017.

González de Vallejo, L.I., (2002). Ingeniería Geológica, PEARSON, Educación, Madrid, $774 \mathrm{pp}$.

Hervas, J; Barredo-Cano, J.I. y Lomoschitz, A.; (2002). "Elaboración de mapas de susceptibilidad de deslizamientos mediante el uso de conjunto de SIG Teledetección y Métodos de Evaluación Multicriterio. Aplicación a la Depresión de Tirajana (Gran Canaria)".

Hervas, J. y Barredo, J.I. (2001). "Evaluación de la susceptibilidad de deslizamientos mediante el uso de conjunto de SIG Teledetección y Métodos de Evaluación Multicriterio. Aplicación de Tirajana (Gran Canaria)".

Instituto Nacional de Estadística, Geografía e Informática (INEGI) (2015). Panorama sociodemográfico de Guerrero, INEGI, 95 pp.

Morán-Zenteno, D. (1984). Geología de la República Mexicana. Secretaría de Programación y Presupuesto, INEGI, $82 \mathrm{pp}$.

Ramos-Bernal, R.N.; Vázquez-Jiménez, R.; Romero-Calcerrada, R.; Novillo, C.J.; Arrogante-Funes, P. y Sánchez-Tizapa, S. (2015). “Identificación de deslizamientos de laderas aplicando técnicas de detección de cambios a imágenes Landsat en la zona costera del Estado de Guerrero, México", Análisis espacial y representación geográfica: innovación y aplicación, Universidad de Zaragoza-AGE, España, pp. 827-834.

Rawat, M.S.; Uniyal, D.P.; Dobhal, R.; Varun, J.; Rawat, B.S.; Bartwal, A.; Devendra, S. and Ashok, A. (2015). "Study of landslide hazard zonation in Mandakini Valley, Rudraprayag district, Uttarakhand using remote sensing and GIS", Current Science, vol. 109, núm. 1, pp. 158-170.

Recondo, C. (2000). "Estudio de las zonas propensas a sufrir deslizamientos en los Concejos de Oviedo y Mieres (Asturias) a partir de una imagen Landsat-TM y de un Modelo Digital de Elevaciones", Revista de Teledetección, vol. 14. 
Romero-Rojas, W.; Ramos-Bernal, R.N.; Vázquez-Jiménez, R.; Arrogante-Funes, P. y Arroyo-Matus, R. (2017). "Detección de deslizamientos de laderas por el método de regresión lineal utilizando imágenes Aster en la zona centro del estado de Guerrero, México", XXV Congreso de la Asociación de Geógrafos Españoles: Naturaleza, Territorio y Ciudad en un Mundo Global, Madrid España, 25-27 de octubre de 2017, pp. 363-372. DOI: <hhttp://doi.org/10.15366/ntc.2017>.

SEDESOL (2013). Catálogo de localidades. Disponible en: $<$ http://www.microrregiones.gob.mx/catloc/LocdeMun.aspx?tipo=clave\&campo $=$ loc\&ent=12\&mun=040>, consultado el 26 de octubre de 2018.

Vázquez-Jiménez, R. (2017). "Método alternativo para la elaboración de cartografía consistente de ocupación y uso del suelo, orientado a estudios de dinámica territorial", tesis de doctorado, Escuela Superior de Ciencias Experimentales y Tecnología, Universidad Rey Juan Carlos, Madrid, España, 297 pp.

Wang, F.; Xu, P.; Wang, C.; Wang, N. y Jiang N. (2017). “Application of a GISbased slope unit method for lanslide susceptibility mapping along the Longzi River, southeastern Tibetan Plateu", International Journal of Geo-Information, China, 20 pp. Disponible en: <http://www.mpdi.com/journal/ijgi6060172>, consultado el 19 de junio de 2017.

Zúñiga-Gutiérrez, M.; Cuevas-Sandoval, A.; Sánchez-Calvo, M. y Barragán-Trinidad, R. (2016). "Análisis de la amenaza de riesgo por deslizamiento de laderas en la ciudad de Chilpancingo, Guerrero", Foro de estudios sobre Guerrero, vol. 2, núm. 3 224-243 pp. 